\title{
ON A SECONDARY INVARIANT OF THE HYPERELLIPTIC MAPPING CLASS GROUP
}

\author{
TAKAYUKI MORIFUJI \\ Department of Mathematics, Tokyo University of Agriculture and Technology \\ 2-24-16 Naka-cho, Koganei, Tokyo 184-8588, Japan \\ E-mail: morifuji@cc.tuat.ac.jp
}

\begin{abstract}
We discuss relations among several invariants of 3-manifolds including Meyer's function, the $\eta$-invariant, the von Neumann $\rho$-invariant and the Casson invariant from the viewpoint of the mapping class group of a surface.
\end{abstract}

1. Introduction. Let $\Sigma_{g}$ be a closed oriented smooth surface of genus $g$ and Diff $+\Sigma_{g}$ the group of orientation preserving diffeomorphisms of $\Sigma_{g}$ equipped with the $C^{\infty}$-topology. The mapping class group $\mathcal{M}_{g}$ is defined to be the group of path components of Diff $+\Sigma_{g}$. In this paper, by an automorphism of $\Sigma_{g}$, we mean an element of $\mathcal{M}_{g}$. Next let $r$ : $\mathcal{M}_{g} \rightarrow \operatorname{Sp}(2 g, \mathbf{Z})$ denote the classical representation defined by the action of $\mathcal{M}_{g}$ on the first integral homology group $H_{1}\left(\Sigma_{g}, \mathbf{Z}\right)$. It is known that $r$ is surjective, and the Torelli group $\mathcal{I}_{g}$ is defined to be the kernel of $r$, so that $\mathcal{I}_{g}$ acts trivially on $H_{1}\left(\Sigma_{g}, \mathbf{Z}\right)$.

Our main object here is the signature cocycle $\tau$, which is a group 2-cocycle of the Siegel modular group $\operatorname{Sp}(2 g, \mathbf{Z})$. It was introduced by Meyer (see [Me]) to describe a signature formula for surface bundles over a surface. If we pull back the cocycle by the representation $r$, then we can think of $\tau$ as a 2-cocycle of $\mathcal{M}_{g}$. We will mainly consider the restriction of $\tau$ to a subgroup of $\mathcal{M}_{g}$, namely, the hyperelliptic mapping class group $\Delta_{g}$, which is the centralizer of a fixed hyperelliptic involution. It is known that $\Delta_{g}=\mathcal{M}_{g}$ if $g=1,2$ and $\Delta_{g} \neq \mathcal{M}_{g}$ if $g \geq 3$. Moreover, the fact that $H^{*}\left(\Delta_{g}, \mathbf{Q}\right)=0$ for $*=1,2$ (see $[\mathrm{C}],[\mathrm{Kw}]$ ) implies that the restriction of $\tau$ to $\Delta_{g}$ must be the coboundary of a unique rational 1-cochain $\phi: \Delta_{g} \rightarrow \mathbf{Q}$ (i.e. $\delta \phi=\left.r^{*} \tau\right|_{\Delta_{g}}$ ). In the following, we call $\phi$ Meyer's function of the hyperelliptic mapping class group. It is a secondary invariant of $\Delta_{g}$ associated with the signature cocycle.

In the case of genus one, explicit formulas of $\tau$ and $\phi$ were given by Meyer himself,

2000 Mathematics Subject Classification: Primary 57R20; Secondary 57M27.

Key words and phrases: Meyer's function, eta-invariant, von Neumann rho-invariant, Casson invariant, Morita's homomorphism.

The paper is in final form and no version of it will be published elsewhere. 
Kirby-Melvin $[\mathrm{KM}]$ and Sczech [Sc]. On the other hand, geometric aspects of Meyer's function have been studied by Atiyah in [A1]. In fact, he related it to various invariants defined for each element of $\Delta_{1}=\mathcal{M}_{1} \cong S L(2, \mathbf{Z})$ including Hirzebruch's signature defect, the logarithmic monodromy of Quillen's determinant line bundle, the Atiyah-PatodiSinger $\eta$-invariant and its adiabatic limit, the special value of the Shimizu $L$-function and so on.

The purpose of this paper is to discuss some extensions of Atiyah's results to higher genus case and explain relations among several invariants of 3-manifolds, such as Meyer's function, the $\eta$-invariant, the von Neumann $\rho$-invariant and the Casson invariant, from the viewpoint of the mapping class group of a surface.

Now we describe the contents of this paper. In the next section, we recall the definitions of the signature cocycle and Meyer's function according to [Me] and [Mf5]. In Section 3 we give a relation between Meyer's function and the $\eta$-invariant under the mapping torus construction. In Section 4 we describe the von Neumann $\rho$-invariant for a $\mathbf{Z}$-covering using Meyer's function. In the last section, the Casson invariant of an integral homology 3 -sphere is described in terms of Meyer's function using the correspondence between elements of $\mathcal{M}_{g}$ and 3-manifolds via Heegaard splitting.

2. Signature cocycle and Meyer's function. In this section, we quickly review the definitions of the signature cocycle and Meyer's function. First we take two elements $A, B \in \operatorname{Sp}(2 g, \mathbf{Z})$ and define the vector space $V_{A, B}$ by

$$
V_{A, B}=\left\{(x, y) \in \mathbf{R}^{2 g} \times \mathbf{R}^{2 g} \mid\left(A^{-1}-I\right) x+(B-I) y=0\right\},
$$

where $I$ denotes the identity matrix. We also define a pairing on $\mathbf{R}^{2 g} \times \mathbf{R}^{2 g}$ by

$$
\left\langle\left(x_{1}, y_{1}\right),\left(x_{2}, y_{2}\right)\right\rangle_{A, B}=\left(x_{1}+y_{1}\right) \cdot J(I-B) y_{2},
$$

where $\cdot$ is the standard inner product in $\mathbf{R}^{2 g}$ and $J$ is the $2 g \times 2 g$ real matrix corresponding to the multiplication by $\sqrt{-1}$ on $\mathbf{C}^{g}=\mathbf{R}^{2 g}$. We can easily check that this pairing is a symmetric bilinear form on $V_{A, B}$ (possibly degenerate), and we define $\tau(A, B)$ to be the signature of the pairing $\langle,\rangle_{A, B}$ on the vector space $V_{A, B}$.

By the Novikov additivity, $\tau(A, B)$ satisfies the so called cocycle condition, namely

$$
\tau(A, B)+\tau(A B, C)=\tau(A, B C)+\tau(B, C),
$$

so that $\tau$ is a group 2-cocycle of the Siegel modular group. It represents the cohomology class $-4 c_{1} \in H^{2}(\mathrm{Sp}(2 g, \mathbf{Z}), \mathbf{Z})$. We call the 2-cocycle $\tau$ the signature cocycle [Me].

REMARK 2.1. (i) The signature cocycle $\tau$ is defined for $\operatorname{Sp}(2 g, \mathbf{Z})$, but we can regard it as a 2-cocycle of $\mathcal{M}_{g}$ in terms of the homology representation $r$. We use the same letter $\tau$ for simplicity. (ii) $\tau(A, B)$ is equal to $\sigma(W)$, the signature of the 4-manifold $W$, which is a surface bundle over the pair of pants with three boundary components $M_{A}, M_{B}$ and $-M_{A B}$, where $M_{A}$ denotes a mapping torus with monodromy $A$. (iii) By definition, $\tau$ is a bounded 2-cocycle. In fact, the range of $|\tau|$ is clearly bounded by $2 g$.

In what follows, we consider the hyperelliptic mapping class group of a surface $\Sigma_{g}$. We fix an involution $\iota \in \mathcal{M}_{g}$ with $2 g+2$ fixed points. The centralizer

$$
\Delta_{g}=\left\{f \in \mathcal{M}_{g} \mid f \iota=\iota f\right\}
$$


is called the hyperelliptic mapping class group of $\Sigma_{g}$ with respect to $\iota$. The conjugacy class of $\Delta_{g}$ in $\mathcal{M}_{g}$ does not depend on the choice of $\iota$.

It was mentioned in the introduction that the rational cohomology groups of $\Delta_{g}$ are trivial for dimensions 1 and 2. Hence the cohomology class represented by $\tau$ is a torsion element in $H^{2}\left(\Delta_{g}, \mathbf{Z}\right)$. More precisely, we can show that the order of $[\tau]$ in $H^{2}\left(\Delta_{g}, \mathbf{Z}\right)$ is $2 g+1($ see $[\mathrm{E}])$. Thus there exists a uniquely defined map

$$
\phi: \Delta_{g} \rightarrow \frac{1}{2 g+1} \mathbf{Z}
$$

such that the coboundary of $\phi$ coincides with the restriction of $\tau$ to $\Delta_{g}$. Here $(1 /(2 g+1)) \mathbf{Z}$ denotes the additive group $\{n /(2 g+1) \in \mathbf{Q} \mid n \in \mathbf{Z}\}$. Namely, we have

$$
\delta \phi\left(f_{1}, f_{2}\right)=\phi\left(f_{2}\right)-\phi\left(f_{1} f_{2}\right)+\phi\left(f_{1}\right)=\tau\left(f_{1}, f_{2}\right)
$$

for $f_{1}, f_{2} \in \Delta_{g}$. We call $\phi$ Meyer's function of the hyperelliptic mapping class group.

REMark 2.2. (i) Meyer's function $\phi$ is a class function of $\Delta_{g}$. That is, for any two elements $f_{1}, f_{2} \in \Delta_{g}$, we have $\phi\left(f_{2} f_{1} f_{2}^{-1}\right)=\phi\left(f_{1}\right)$. Consequently, we can regard $\phi$ as an invariant of surface bundles over the circle. (ii) The relation $\delta \phi=\left.\tau\right|_{\Delta_{g}}(g \geq 2)$ implies that $\phi$ is a homomorphism on the Torelli group $\mathcal{I}_{g} \cap \Delta_{g}$, because $\tau$ is originally defined on the Siegel modular group $\operatorname{Sp}(2 g, \mathbf{Z})$.

The group presentation of $\Delta_{g}$ was given by Birman and Hilden (see $[\mathrm{BH}]$ ). By using its defining relations, we can determine the values of $\phi$ on generators of $\Delta_{g}$.

EXAMPLE 2.3. For the generators $\zeta_{1}, \zeta_{2}, \ldots, \zeta_{2 g+1}$ of $\Delta_{g}(g \geq 2)$, the values of Meyer's function $\phi$ are equal to $(g+1) /(2 g+1)$. This is shown as follows. First each generator is mutually conjugate (in fact, $\zeta_{i+1}=\xi \zeta_{i} \xi^{-1}$ holds for $\xi=\zeta_{1} \cdots \zeta_{2 g+1}$ ), so that $\phi$ has the same value on generators. Then substituting a defining relation of $\Delta_{g}$ for $\delta \phi=\tau$, we can evaluate $\phi\left(\zeta_{i}\right)$ explicitly.

EXAMPLE 2.4. Let $\psi_{h} \in \Delta_{g}(1 \leq h \leq g-1)$ be a BSCC-map of genus $h$, that is, a Dehn twist along a bounding simple closed curve on $\Sigma_{g}$ which is invariant under the action of the hyperelliptic involution $\iota$ and which separates $\Sigma_{g}$ into two subsurfaces of genera $h$ and $g-h$. Then $\psi_{h}$ is presented by $\psi_{h}=f\left(\zeta_{1} \cdots \zeta_{2 h}\right)^{4 h+2} f^{-1}$ for some automorphism $f \in \Delta_{g}$. Thus we have $\phi\left(\psi_{h}\right)=-4 h(g-h) /(2 g+1)$.

REMARK 2.5. (i) There exist other constructions of Meyer's function for genus 2, which are due to Kasagawa [Kg] and Iida [I]. (ii) Meyer's function plays an important role in the study of certain singular fibrations. In fact, Matsumoto [Ma] and Endo [E] defined the local signature of hyperelliptic Lefschetz fibrations by using Meyer's function. See also $[\mathrm{Ku}]$ for Meyer's function of plane curves.

3. Eta-invariant. In this section, we discuss a relation between Meyer's function and the $\eta$-invariant of a surface bundle over the circle. Let $M$ be an oriented closed Riemannian 3 -manifold. By using the spectrum of the signature operator, the $\eta$-invariant of $M$ is defined to be the value at zero of the $\eta$-function associated with the signature operator. Instead of giving the precise definition of the $\eta$-invariant, we recall the following index theorem due to Atiyah, Patodi and Singer (see [APS]). Let $W$ be a compact oriented 
Riemannian 4-manifold with product metric near the boundary $\partial W=M$. Then the $\eta$-invariant of $M$ is given by

$$
\eta(M)=\frac{1}{3} \int_{W} p_{1}-\sigma(W)
$$

where $p_{1}$ is the first Pontrjagin form for the Riemannian connection on the tangent bundle $T W$ of $W$. It is well-known that, if $W$ is a closed 4-manifold, then the signature $\sigma(W)$ is described by the integral of $p_{1}$. Therefore, the above formula can be viewed as an extension of the Hirzebruch signature formula to 4-manifold with boundary. Note that the $\eta$-invariant is not a topological but rather a spectral invariant.

Now for an automorphism $f \in \mathcal{M}_{g}$, let $M_{f}$ be the mapping torus corresponding to $f$. Namely, it is the identification space $\Sigma_{g} \times \mathbf{R} /(x, t) \sim(f(x), t+1)$. When $f \in \mathcal{M}_{g}$ is of finite order (or a periodic automorphism), we endow $M_{f}$ with the metric which is induced from the product of the standard metric on the circle and an $f$-invariant metric of $\Sigma_{g}$. If we restrict ourselves to the hyperelliptic mapping class group $\Delta_{g}$, we obtain the following theorem.

THEOREM 3.1. $\eta\left(M_{f}\right)=\phi(f)$ for any periodic automorphism $f \in \Delta_{g}$.

This theorem is proved with the help of an explicit formula for the $\eta$-invariant of a periodic automorphism of the mapping class group $\mathcal{M}_{g}$ (see [Mf1]). More precisely,

$$
\eta\left(M_{f}\right)=\frac{1}{n} \sum_{k=1}^{n-1} \tau\left(f, f^{k}\right)
$$

for $f \in \mathcal{M}_{g}$ of the order $n$. The formula is based on the index theorem, due to Atiyah, Patodi and Singer, and uses the notion of 2 -framing on a 3 -manifold, which is a trivialization of twice the tangent bundle $2 T M=T M \oplus T M$ as a $\operatorname{Spin}(6)$-bundle (see [A2] for details).

EXAMPLE 3.2. We can explicitly calculate all the values of Meyer's function $\phi$ on periodic automorphisms for the genus 1 and 2 cases by using the homological action of each periodic automorphism (see [Mf2]). In particular, we notice a relation to the NielsenThurston theory of surface automorphisms [CB]. It is known that the theory classifies the automorphisms of $\Sigma_{g}$ into the following three types: (i) periodic, (ii) reducible and (iii) pseudo-Anosov. We say that an automorphism of $\Sigma_{g}$ is reducible if it has a representative which leaves some essential 1-submanifold of the surface invariant. We then easily see that (i) and (ii) have some overlap, although (iii) cannot have any intersection with either of them. Hence a given periodic automorphism is either reducible or irreducible. In fact, there are several works concerning the characterization of the reducibility of periodic automorphisms (see [Kh], [Mf3] and references therein).

From the direct computation of Meyer's function in the genus 1 or 2 cases, we see that a periodic automorphism $f$ is reducible if and only if $\phi(f)=0$ (i.e. $\eta\left(M_{f}\right)=$ $0)$. Thus we can say that the reducibility of periodic automorphisms of a surface with low genus is characterized by the vanishing of Meyer's function or the $\eta$-invariant. The above statement need not hold for higher genera, but we can obtain a similar result for automorphisms of prime order of hyperelliptic Riemann surfaces (see [Mf4]). 
As an immediate corollary of Theorem 3.1, we have the following result.

Corollary 3.3. Let $f \in \mathcal{M}_{g}$ be a periodic automorphism. If $f \in \Delta_{g}$, that is, $f$ commutes with the fixed hyperelliptic involution $\iota$, then $\eta\left(M_{f}\right) \in(1 /(2 g+1)) \mathbf{Z}$.

Thus in some sense, we may regard the $\eta$-invariant as an obstruction to a periodic automorphism being hyperelliptic.

EXAMPLE 3.4. Let $f \in \mathcal{M}_{3}$ be a periodic automorphism of order 3 so that the quotient orbifold of $\Sigma_{3}$ by its cyclic action is homeomorphic to $S^{2}(3,3,3,3,3)$ (2-sphere with five cone points of index 3 ). Then an easy computation shows that the $\eta$-invariant of the corresponding mapping torus is given by

$$
\eta\left(M_{f}\right)=-\frac{2}{3} \notin \frac{1}{7} \mathbf{Z} .
$$

Hence, Corollary 3.3 implies that $f$ cannot commute with the hyperelliptic involution $\iota$.

REMARK 3.5. Let $G$ be the finite cyclic group generated by a periodic automorphism $f \in \mathcal{M}_{g}$. Since $H^{*}(G, \mathbf{Q})=0$ for $*=1,2$, we can define Meyer's function $\phi_{G}: G \rightarrow \mathbf{Q}$ of $G$. As an analogue of Theorem 3.1, we see that $\eta\left(M_{f}\right)=\phi_{G}(f)$ for any periodic automorphism $f \in \mathcal{M}_{g}$ (see $[\mathrm{Ak}]$ ).

4. The von Neumann rho-invariant. Let $\Gamma$ be a discrete group and $M$ an oriented closed Riemannian 3-manifold. In addition, we assume that we are given a surjective homomorphism from the fundamental group $\pi_{1} M$ to $\Gamma$. Then we can take a $\Gamma$-covering $\Gamma \rightarrow \hat{M} \rightarrow M$. Lifting the metric and the signature operator to $\hat{M}$, we can define the $\eta$-invariant of $\hat{M}$, denoted by $\eta^{(2)}(\hat{M})$. We call it the von Neumann $\eta$-invariant. It is also known that $\eta^{(2)}(\hat{M})$ fits into the index theorem.

The $\eta$-invariant and the von Neumann $\eta$-invariant depend on the Riemannian metric, but Cheeger-Gromov showed in [CG] that their difference is independent of the Riemannian metric. We denote the difference $\eta^{(2)}(\hat{M})-\eta(M)$ by $\rho^{(2)}(\hat{M})$ and call it the von Neumann $\rho$-invariant.

In some sense, the invariant $\rho^{(2)}$ is an extension of the classical $\rho$-invariant of Atiyah, Patodi and Singer : the difference between the $\eta$-invariant $\eta_{\gamma}$ twisted by a unitary representation $\gamma: \pi_{1} M \rightarrow U(n)$ and the original $\eta$-invariant does not depend on the choice of the Riemannian metric either.

Given an automorphism $f \in \Delta_{g}$, consider the $\mathbf{Z}$-covering $\mathbf{Z} \rightarrow \hat{M}_{f} \rightarrow M_{f}$ associated with a surjective homomorphism $\pi_{1} M_{f} \rightarrow \pi_{1} S^{1} \cong \mathbf{Z}$. One can easily see that Meyer's function is not multiplicative with respect to coverings. However, it is related to the von Neumann $\rho$-invariant after passing to a limit. Namely, we have the following result.

THEOREM 4.1. $\rho^{(2)}\left(\hat{M}_{f}\right)=\lim _{k \rightarrow \infty} \frac{\phi\left(f^{k}\right)-k \phi(f)}{k}$.

To be more precise, the von Neumann $\rho$-invariant $\rho^{(2)}\left(\hat{M}_{f}\right)$ is the limit of the deviation from the multiplicativity of $\phi$ for a finite covering $M_{f^{k}} \rightarrow M_{f}$. This is shown by using Propositions 2.2 and 3.1 in [Mf1] and the approximation theorem for the $\eta$-invariant, due 
to Vaillant [V] and Lück-Schick [LS], which states that

$$
\eta^{(2)}(\hat{M})=\lim _{k \rightarrow \infty} \frac{\eta\left(M_{(k)}\right)}{\left[\Gamma: \Gamma_{k}\right]}
$$

for a descending sequence of normal subgroups $\Gamma \supset \Gamma_{1} \supset \Gamma_{2} \supset \cdots$ such that $\left[\Gamma: \Gamma_{k}\right]<\infty$ and $\cap_{k} \Gamma_{k}=\{1\}$, and $\Gamma / \Gamma_{k}$-covering $M_{(k)}=\hat{M} / \Gamma_{k} \rightarrow M$.

EXAMPLE 4.2. As an example, we consider the genus one case. In this case, all elements $A \in S L(2, \mathbf{Z})$ fall into the following three categories:

(i) Elliptic case: $|\operatorname{tr} A|<2$. Let $A_{n} \in S L(2, \mathbf{Z})$ have the order $n(n=3,4,6)$. We can take

$$
A_{3}=\left(\begin{array}{cc}
-1 & -1 \\
1 & 0
\end{array}\right), \quad A_{4}=\left(\begin{array}{cc}
0 & -1 \\
1 & 0
\end{array}\right) \quad \text { and } \quad A_{6}=\left(\begin{array}{cc}
0 & -1 \\
1 & 1
\end{array}\right) \text {. }
$$

An easy calculation shows that

$$
\begin{gathered}
\phi\left(A_{3}\right)=-\phi\left({A_{3}}^{2}\right)=-2 / 3, \phi\left(A_{4}\right)=-\phi\left(A_{4}{ }^{3}\right)=-1, \phi\left(A_{4}{ }^{2}\right)=0, \\
\phi\left(A_{6}\right)=-\phi\left(A_{6}{ }^{5}\right)=-4 / 3, \phi\left({A_{6}}^{2}\right)=-\phi\left(A_{6}{ }^{4}\right)=-2 / 3, \phi\left(A_{6}{ }^{3}\right)=0 .
\end{gathered}
$$

Needless to say, $\phi\left(A_{n}{ }^{n}\right)=\phi(I)=0$. Hence we have

$$
\rho^{(2)}\left(\hat{M}_{A_{n}}\right)= \begin{cases}2 / 3, & n=3 \\ 1, & n=4 \\ 4 / 3, & n=6\end{cases}
$$

in terms of Theorem 4.1. It should be noted that $\rho^{(2)}\left(\hat{M}_{f}\right)=0$ for any involution $f \in \mathcal{M}_{g}$ (see [Mf1], [Mf6]).

(ii) Parabolic case: $|\operatorname{tr} A|=2$. We can take $A_{b}=\left(\begin{array}{ll}1 & b \\ 0 & 1\end{array}\right)(b \in \mathbf{Z})$. Then we obtain $\rho^{(2)}\left(\hat{M}_{A_{b}}\right)=-\operatorname{sgn}(b)$, where $\operatorname{sgn}(b)=b /|b|$ if $b \neq 0$ and 0 if $b=0$. This follows from Atiyah's calculation in [A1].

(iii) Hyperbolic case : $|\operatorname{tr} A|>2$. Since Meyer's function of genus one satisfies $\phi\left(A^{k}\right)=$ $k \phi(A)$ for a hyperbolic element $A \in S L(2, \mathbf{Z})$ (see $[\mathrm{Me}]$ ), we have $\rho^{(2)}\left(\hat{M}_{A}\right)=0$ by virtue of Theorem 4.1.

An immediate corollary of Theorem 4.1 and Remark 2.2 (ii) is the following result for the higher genus case.

Corollary 4.3. If $f$ is an automorphism in $\mathcal{I}_{g} \cap \Delta_{g}$, then $\rho^{(2)}\left(\hat{M}_{f}\right)=0$.

Here we wish to make a remark. If we restrict the above theorem to the level 2 subgroup of $\mathcal{M}_{g}$, we can describe a relation among the von Neumann $\rho$-invariant, the first Morita-Mumford class $e_{1} \in H^{2}\left(\mathcal{M}_{g}, \mathbf{Z}\right)$ (see [Mt1], [Mu] and Section 5 below) and the Rokhlin invariant of a spin 3-manifold in the framework of the bounded cohomology [G]. Roughly speaking, the pull-back of $e_{1}$ into $H_{b}^{2}\left(S^{1}, \mathbf{Z}\right) \cong \mathbf{R} / \mathbf{Z}$ via a holonomy homomorphism is given by the linear combination of the Rokhlin invariant and the von Neumann $\rho$-invariant (see [Ki2], [Mf6] for details). We describe it more explicitly in the appendix to this paper.

5. Casson invariant. In this last section, we explain a relation between Meyer's function and the Casson invariant. The Casson invariant $\lambda(M)$ is an integer valued invariant 
defined for an oriented integral homology 3 -sphere $M$. Roughly speaking, it is the number of conjugacy classes of irreducible representations of the fundamental group $\pi_{1} M$ into the Lie group $S U(2)$.

On the other hand, from the theory of characteristic classes of surface bundles, due to Morita (see [Mt2], [Mt3]), the Casson invariant $\lambda$ can be interpreted as a secondary invariant associated with the first Morita-Mumford class $e_{1}$, using the correspondence between elements of the mapping class group and 3-manifolds via Heegaard splitting.

Let us state this more precisely. We define $\mathcal{K}_{g}$ to be the subgroup of $\mathcal{M}_{g}$ generated by Dehn twists along bounding simple closed curves on $\Sigma_{g}$. This is also a subgroup of the Torelli group $\mathcal{I}_{g}$, because clearly, the action of a generator of $\mathcal{K}_{g}$ on the homology of $\Sigma_{g}$ is trivial. Next we fix a Heegaard splitting of the 3 -sphere $S^{3}=\mathcal{H}_{g} \cup_{\iota_{g}}-\mathcal{H}_{g}$, where $\mathcal{H}_{g}$ denotes the handlebody of genus $g$ and $\iota_{g}$ is the gluing map for this splitting.

For an automorphism $f \in \mathcal{K}_{g}$, we construct a 3 -manifold $M^{f}$ by regluing two handlebodies via the composition $\iota_{g} f$. Then it is easy to see that the resulting manifold $M^{f}$ is again a homology 3-sphere, because $f$ acts on $H_{1}\left(\Sigma_{g}, \mathbf{Z}\right)$ trivially. Thereby we can evaluate its Casson invariant $\lambda\left(M^{f}\right)$. In short, Morita's results claim that there exists a homomorphism $\lambda^{*}: \mathcal{K}_{g} \rightarrow \mathbf{Z}$ such that $\lambda^{*}(f)=\lambda\left(M^{f}\right)$. More precisely, $\lambda^{*}$ consists of the sum of two homomorphisms. One is Morita's homomorphism $d_{0}: \mathcal{K}_{g} \rightarrow \mathbf{Q}$, which is at the core of the Casson invariant theory from the viewpoint of the mapping class group. The other is the Johnson homomorphism, which can be expressed in terms of the higher Massey products of mapping tori (see [Ki1]).

Now assume that $g \geq 2$. We then have the following result.

THEOREM 5.1. Meyer's function essentially coincides with Morita's homomorphism on $\mathcal{K}_{g} \cap \Delta_{g}$. To be more precise,

$$
\phi(f)=\frac{1}{3} d_{0}(f)
$$

for any automorphism $f \in \mathcal{K}_{g} \cap \Delta_{g}$.

Therefore, in principle, we can say that the Casson invariant of an integral homology 3 -sphere is determined by Meyer's function. Here, as an example, we evaluate Morita's homomorphism on a typical element in $\mathcal{K}_{g} \cap \Delta_{g}$.

EXAMPLE 5.2. Let $\psi_{h} \in \mathcal{K}_{g} \cap \Delta_{g}$ be a BSCC-map of genus $h$. Then the value of Morita's homomorphism on $\psi_{h}$ is given by

$$
d_{0}\left(\psi_{h}\right)=-\frac{12}{2 g+1} h(g-h)
$$

using Theorem 5.1 and Example 2.4.

Finally let us briefly review the definition of Morita's homomorphism. It is a secondary invariant associated with the first Morita-Mumford class $e_{1} \in H^{2}\left(\mathcal{M}_{g}, \mathbf{Z}\right)$.

Let $e \in H^{2}\left(\mathcal{M}_{g, *}, \mathbf{Z}\right)$ be the Euler class of the central $\mathbf{Z}$-extension $\mathbf{Z} \rightarrow \mathcal{M}_{g, 1} \rightarrow \mathcal{M}_{g, *}$, where $\mathcal{M}_{g, 1}$ and $\mathcal{M}_{g, *}$ denote the mapping class groups of $\Sigma_{g}$ relative to an embedded disc $D \subset \Sigma_{g}$ and a base point $* \in D$ respectively. The center $\mathbf{Z}$ is generated by the Dehn twist parallel to $\partial D$. We define $e_{1}$ to be the Gysin image (integration along the fiber) of $e^{2}$. This is an element of $H^{2}\left(\mathcal{M}_{g}, \mathbf{Z}\right)$; it is called the first Morita-Mumford class. 
Now it is known that there exist two canonical 2-cocycles over $\mathbf{Q}$ representing $e_{1}$. One is the signature cocycle $\tau\left(e_{1}=[-3 \tau]\right)$ and the other is the intersection cocycle $c$ (the latter one is defined once we fix a certain crossed homomorphism of $\mathcal{M}_{g}$ ). Therefore, we have the uniquely defined mapping $d: \mathcal{M}_{g} \rightarrow \mathbf{Q}$ so that $\delta d=c+3 \tau$. The uniqueness follows from the fact that $\mathcal{M}_{g}$ is perfect for $g \geq 3$. We denote the restriction of $d$ to the subgroup $\mathcal{K}_{g}$ by $d_{0}$. Morita showed that $d_{0}$ does not depend on the choice of the crossed homomorphism, and it serves as a generator of $H^{1}\left(\mathcal{K}_{g}, \mathbf{Z}\right)^{\mathcal{M}_{g}}$. This $d_{0}$ is the map that we have called Morita's homomorphism.

The key to the proof of Theorem 5.1 is to construct a crossed homomorphism of $\mathcal{M}_{g}$ so that the restriction of it to the hyperelliptic mapping class group $\Delta_{g}$ is zero. Using it to define the intersection cocycle, we can obtain a relation between Meyer's function and Morita's homomorphism.

REMARK 5.3. In [Mt4], Morita gave an interpretation of $d_{0}$ in terms of Hirzebruch's signature defect of certain framed 3-manifolds. Combining it and Theorem 5.1, we have a generalization of Atiyah's result mentioned in the introduction.

Appendix. We give here a description of the first Morita-Mumford class $e_{1}$ via the Rokhlin invariant and the von Neumann $\rho$-invariant in the bounded cohomology $H_{b}^{*}$.

First, note that $e_{1}$ is a bounded cohomology class (see Remark 2.1 (iii)) and consider $e_{1}$ on $\mathcal{M}_{g, *}$ rather than on $\mathcal{M}_{g}$ for technical reasons. The pull back of $e_{1}$ via the holonomy homomorphism $f: \pi_{1} S^{1} \rightarrow \mathcal{M}_{g, *}$ of a surface bundle over the circle clearly vanishes because $H^{2}\left(S^{1}, \mathbf{Z}\right)=0$. However, as Kitano showed in [Ki2], the class $e_{1} / 48$, which depends on the spin structure of $\Sigma_{g}$, makes sense as a bounded cohomology class in $H_{b}^{2}\left(S^{1}, \mathbf{Z}\right) \cong \mathbf{R} / \mathbf{Z}$, and it is given by the Rokhlin invariant $\mu$ if the image of $f$ is contained in the Torelli group $\mathcal{I}_{g, *}$. Combining Theorem 4.1 with the result of Kitano, we have the following on the level 2 subgroup $\mathcal{M}_{g, *}(2)=\operatorname{Ker}\left\{\mathcal{M}_{g, *} \rightarrow\right.$ $\operatorname{Sp}(2 g, \mathbf{Z} / 2)\} \supset \mathcal{I}_{g, *}:$ for $f: \mathbf{Z} \rightarrow \mathcal{M}_{g, *}(2)$, the pull-back $f^{*} e_{1} / 48 \in H_{b}^{2}(\mathbf{Z}, \mathbf{Z})$ is represented by $\mu\left(M_{f}, \tilde{\alpha}\right)-\rho^{(2)}\left(\hat{M}_{f}\right) / 16 \in H^{1}(\mathbf{Z}, \mathbf{R} / \mathbf{Z})$. The correspondence between them is given by the isomorphism $H_{b}^{2}(\mathbf{Z}, \mathbf{Z}) \cong H_{b}^{1}(\mathbf{Z}, \mathbf{R} / \mathbf{Z}) \cong H^{1}(\mathbf{Z}, \mathbf{R} / \mathbf{Z})$.

Let us briefly review the definition of the Rokhlin invariant. Let $(M, \alpha)$ be an oriented spin 3-manifold with a spin structure $\alpha$. Then there exists a compact oriented spin 4manifold $(W, \beta)$ such that $\partial W=M$ and $\left.\beta\right|_{M}=\alpha$. The Rokhlin invariant $\mu(M, \alpha) \in \mathbf{Q} / \mathbf{Z}$ is defined by

$$
\mu(M, \alpha)=\frac{\sigma(W)}{16} \bmod \mathbf{Z} .
$$

It does not depend on the choice of the 4-manifold $W$ by virtue of Rokhlin's theorem.

According to Miller and Lee [ML], the Rokhlin invariant of a spin 3-manifold is a spectral invariant. To be more precise, it is described by the $\eta$-invariants as follows. Let $W, M$ be as in Section 3 and assume further that $W$ (hence also $M$ ) is a spin manifold. Let $\mathcal{D}$ denote the Dirac operator of $M$ acting on the spinor fields. This is a self-adjoint elliptic operator, so that the $\eta$-invariant $\eta_{\mathcal{D}}(M)$ is defined. We then have

$$
\operatorname{ind}(\mathcal{D})=-\frac{1}{24} \int_{W} p_{1}-\frac{1}{2}\left\{\hbar+\eta_{\mathcal{D}}(M)\right\},
$$


where $\hbar$ is the dimension of the space of harmonic spinors on $M$. Combining the above formula and the index theorem mentioned in Section 3, we get

$$
\sigma(W)+8 \operatorname{ind}(\mathcal{D})=-\eta(M)-4\left\{\hbar+\eta_{\mathcal{D}}(M)\right\} .
$$

The basic spin representations $\mathcal{S}^{ \pm}$of $\operatorname{Spin}(4)$ are quaternionic and hence the index of the Dirac operator is always even. We therefore obtain

$$
\mu(M, \alpha)=-\frac{1}{16} \eta(M)-\frac{1}{4}\left\{\hbar+\eta_{\mathcal{D}}(M)\right\} \quad \bmod \mathbf{Z}
$$

which shows that the Rokhlin invariant is a spectral invariant.

Let us fix a spin structure $\alpha$ of $\Sigma_{g}(g \geq 2)$. For any automorphism $f \in \mathcal{M}_{g, *}(2)$, there exists a uniquely defined spin structure $\tilde{\alpha}$ on $M_{f}$ whose restriction to each fiber is $\alpha$, and whose restriction to the $S^{1}$-orbit of $* \in \Sigma_{g}$ is the bounding spin structure. Applying the above formula to $\left(M_{f}, \tilde{\alpha}\right)$ and substituting it for our description of the first Morita-Mumford class, we conclude that

$$
f^{*} e_{1} / 48=-\frac{1}{16} \eta^{(2)}\left(\hat{M}_{f}\right)-\frac{1}{4}\left\{\hbar+\eta_{\mathcal{D}}\left(M_{f}\right)\right\} \quad \bmod \mathbf{Z}
$$

for any automorphism $f \in \mathcal{M}_{g, *}(2)$.

Acknowledgments. This research was supported by the Grant-in-Aid for Scientific Research (No. 17740032), the Ministry of Education, Culture, Sports, Science and Technology, Japan.

\section{References}

[Ak] T. Akita, On the $\eta$-invariant of mapping tori of closed surfaces, 1998 (in Japanese).

[A1] M. F. Atiyah, The logarithm of the Dedekind $\eta$-function, Math. Ann. 278 (1987), 335380.

[A2] M. F. Atiyah, On framings of 3-manifolds, Topology 29 (1990), 1-7.

[APS] M. F. Atiyah, V. K. Patodi and I. M. Singer, Spectral asymmetry and Riemannian geometry I, Math. Proc. Cambridge Philos. Soc. 78 (1975), 43-69.

[BH] J. Birman and H. Hilden, On mapping class groups of closed surfaces as covering spaces, Advances in the theory of Riemann surfaces, 66, Princeton Univ. Press, 1971, 81-115.

[CB] A. J. Casson and S. A. Bleiler, Automorphisms of surfaces after Nielsen and Thurston, Cambridge Univ. Press, 1988.

[CG] J. Cheeger and M. Gromov, Bounds on the von Neumann dimension of $L^{2}$-cohomology and the Gauss-Bonnet theorem for open manifolds, J. Differential Geom. 21 (1985), $1-34$.

[C] F. R. Cohen, Homology of mapping class groups for surfaces of low genus, in: Contemp. Math. 58, Amer. Math. Soc., 1987, 21-30.

[E] H. Endo, Meyer's signature cocycle and hyperelliptic fibrations, Math. Ann. 316 (2000), 237-257.

[G] M. Gromov, Volume and bounded cohomology, Inst. Hautes Études Sci. Publ. Math. 56 (1982), 5-100.

[I] S. Iida, Adiabatic limits of $\eta$-invariants and the Meyer function for smooth theta divisors, RIMS Kokyuroku 1490 (2006), 109-126. 
[Kg] R. Kasagawa, On a function on the mapping class group of a surface of genus 2, Topology Appl. 102 (2000), 219-237.

[Kh] Y. Kasahara, Reducibility and orders of periodic automorphisms of surfaces, Osaka J. Math. 28 (1991), 985-997.

[Kw] N. Kawazumi, Homology of hyperelliptic mapping class groups for surfaces, Topology Appl. 76 (1997), 203-216.

[KM] R. Kirby and P. Melvin, Dedekind sums, $\mu$-invariants and the signature cocycle, Math. Ann. 299 (1994), 231-267.

[Ki1] T. Kitano, Johnson's homomorphisms of subgroups of the mapping class group, the Magnus expansion and Massey higher products of mapping tori, Topology Appl. 69 (1996), 165-172.

[Ki2] T. Kitano, On the first Morita-Mumford class of surface bundles over $S^{1}$ and the Rokhlin invariant, J. Knot Theory Ramifications 9 (2000), 179-186.

$[\mathrm{Ku}] \quad \mathrm{Y}$. Kuno, The mapping class group and the Meyer function for plane curves, Math. Ann. 342 (2008), 923-949.

[LS] W. Lück and T. Schick, Approximating $L^{2}$-signatures by their finite-dimensional analogues, Forum Math. 17 (2005), 31-65.

[Ma] Y. Matsumoto, Lefschetz fibrations of genus two-a topological approach, in: Topology and Teichmüller Spaces (Katinkulta, 1995), World Sci. Publ., River Edge, NJ, 1996, 123-148.

[Me] W. Meyer, Die Signatur von Flächenbündeln, Math. Ann. 201 (1973), 239-264.

[ML] E. Y. Miller and R. Lee, Some invariants of spin manifolds, Topology Appl. 25 (1987), 301-311.

[Mf1] T. Morifuji, The $\eta$-invariant of mapping tori with finite monodromies, Topology Appl. 75 (1997), 41-49.

[Mf2] T. Morifuji, On the reducibility and the $\eta$-invariant of periodic automorphisms of genus 2 surface, J. Knot Theory Ramifications 6 (1997), 827-831.

[Mf3] T. Morifuji, A note on the reducibility of automorphisms of the Klein curve and the $\eta$-invariant of mapping tori, Proc. Amer. Math. Soc. 126 (1998), 1945-1947.

[Mf4] T. Morifuji, A remark on the $\eta$-invariant for automorphisms of hyperelliptic Riemann surfaces, Bull. Austral. Math. Soc. 62 (2000), 177-182.

[Mf5] T. Morifuji, On Meyer's function of hyperelliptic mapping class groups, J. Math. Soc. Japan 55 (2003), 117-129.

[Mf6] T. Morifuji, A note on von Neumann rho-invariant of surface bundles over the circle, Tohoku Math. J. 58 (2006), 123-127.

[Mt1] S. Morita, Characteristic classes of surface bundles, Invent. Math. 90 (1987), 551-577.

[Mt2] S. Morita, Casson's invariant for homology 3-spheres and characteristic classes of surface bundles, Topology 28 (1989), 305-323.

[Mt3] S. Morita, On the structure of the Torelli group and the Casson invariant, Topology 30 (1991), 603-621.

[Mt4] S. Morita, Casson invariant, signature defect of framed manifolds and the secondary characteristic classes of surface bundles, J. Differential Geom. 47 (1997), 560-599.

$[\mathrm{Mu}]$ D. Mumford, Towards an enumerative geometry of the moduli space of curves, in: Arithmetic and Geometry, Progr. Math. 36, Birkhäuser, 1983, 271-328.

[Sc] R. Sczech, Dedekind sums and signatures of intersection forms, Math. Ann. 299 (1994), 269-274.

[V] B. Vaillant, Indextheorie für Überlagerungen, Diplomarbeit, Universität Bonn, 1997. 